\title{
A Study on the Life World of Chinese Elders with Assisted Caring by Institutions: From the Perspective of Ethnographic Psychology
}

\author{
Tangsheng Wang ${ }^{1}$,, Siyu Qian ${ }^{2}$, Yabei Zeng ${ }^{3}$ \\ ${ }^{1}$ School of Philosophy, Wuhan University, Wuhan City, China \\ ${ }^{2}$ Affiliated High School, South China Normal University, Guangzhou City, China \\ ${ }^{3}$ Chongqing Nankai Secondary School, Chongqing City, China

\section{Email address:} \\ wangtangsheng@whu.edu.cn (Tangsheng Wang), qsysiyu@163.com (Siyu Qian),2242694729@qq.com (Yabei Zeng) \\ ${ }^{*}$ Corresponding author
}

\section{To cite this article:}

Tangsheng Wang, Siyu Qian, Yabei Zeng. A Study on the Life World of Chinese Elders with Assisted Caring by Institutions: From the Perspective of Ethnographic Psychology. Psychology and Behavioral Sciences. Vol. 7, No. 3, 2018, pp. $62-68$.

doi: $10.11648 /$ j.pbs.20180703.14

Received: August 7, 2018; Accepted: September 4, 2018; Published: October 11, 2018

\begin{abstract}
The global society is gradually paying more and more attention to the elders with the rapidly ageing population in the world, and Chinese researchers pay more attention to how to care for the elders. The current study focuses on the elders living in a nursing home and living at home with community assistance. Employing ethnographic methods to study aging psychology, researchers interviewed 21 elders to analyze the focus in their life world during two year of participatory observation, meanwhile the physical and social environments definite sample attributes of elders with assisted caring by institutions. The result shows that although the backgrounds and personalities of these group members were different, they talked about common topics such as age, health, family relationships, neighborhood relationships, incomes, life feelings, and life strategies. The common theme was how to live a good life, including that Living in Nursing Home to Adapt to Frailty in Body, the Life in the Nursing Home is a continuous form of the Life at Home, Keeping positive to live a Good Life and believing that Longevity Is a Blessing. Either silent behaviors or voiced conversations are showing the obvious meaning of life. In a modern society with advanced technology causing elders to be isolated, this is thought-provoking.
\end{abstract}

Keywords: Life World, Assisted Caring by Institutions, Elders, Ethnographic Psychology

\section{Introduction}

Currently, the global society is gradually paying more and more attention to the elders with the rapidly ageing population in the world. According to the Madrid Political Declaration of United Nations [1], by 2050, the number of world population of elders over 60 years old will increase from six hundred million to approximately two billion; the proportion of elders over 60 in the world population is expected to double, from $10 \%$ to $21 \%$; the developing countries will have the most significant and the fastest increase. In addition, by 2050 , it is estimated that the proportion of elders over 60 in Chinese population will be $36.5 \%$, which is nearly 492 million people; elders over 80 years old will account for $8.9 \%$ in Chinese population, which is approximately 120 million people [2]. In other words, during the upcoming 35 years, elders over 60 years old will double, and elders over 80 will more than quadruple.

According to previous research, comparing to western researchers who focus more on how to maintain elders' good mental health conditions, Chinese researchers pay more attention to how to care for the elders [3]. Therefore, how does China solve the caring problem of the elders currently?

Shen Ke studied about the change of living mode of old people and considered nursing home as a forced choice for the elders to live in: the family members of the elders with poor self-care ability might only give them limited care; their families might have limited living space; or elders might frequently have disagreements with their kids when living together [4]. Currently, the distribution of nursing institutions for the elders is unbalanced: 
good nursing homes centralize in cities and towns; because of their low pricing and good service, public nursing homes with government support are too popular to have vacancy to accommodate more elders; good private nursing homes, however, often price high due to lack of government funding. For this reason, elders with limited incomes can hardly live in a satisfying nursing home. By 2008, it is estimated that $3.5 \%$ of the elders are provided for in nursing institutions located in cities and towns and $0.8 \%$ in nursing institutions located in countryside.

Based on the growth of proportion of nursing home beds per thousand old people have by 2016 in China [5, 6] and the proportion of elders provided for in nursing institutions [7], the number of increased nursing home beds is more than that of elders accommodated, so many vacancies exist in nursing institutions. The increase in nursing home beds does not influence the ratio of the elders provided for in nursing homes. Although the number of elders in nursing homes is constantly increasing, the total number of old people almost always accounts for approximately $70 \%$ of nursing home beds, which indicates that the supply of nursing homes is not the main factor in influencing whether or not the elders choose this living mode.

Living modes often have different impacts on the self-care ability, health, and life satisfaction of the elders. Comparing with living alone, living with their kids will have negative impact on self-care ability and cognition but positive impact on anti-depression and life satisfaction. Whether or not receiving relatively satisfying incomes, whether or not receiving timely medication when in poor health condition, and whether or not having someone to talk to all play important roles(control/mediate) in old people's cognition, self-evaluated health condition, and life satisfaction [4].

According to research conducted by Zeng Yi et al. [7], lacking people to care for the elders is the main reason why old people choose to live in nursing homes. Among the investigated old people who live in nursing homes and have poor mobility, nearly $60 \%$ of them choose to live in nursing homes because they do not have kids or their kids cannot take care of them; $26 \%$ of them do not want to be the burden for their kids; $9 \%$ of them want to communicate with other old people; $3 \%$ of them do not have their own houses and do not want to live with their kids.

The study of Lin Xianming and Liu Yongce preliminarily discusses the influences of being provided for at home or in nursing homes on life satisfaction of the elders. First, whether or not old people live in nursing homes voluntarily has significant influence on the sense of loneliness among the elders in nursing homes $(p=0.010)$, and it also influences the cultural activities and interpersonal relationships of the elders $(p=0.006)$. Second, the sense of loneliness does not differ significantly between being provided for at home or in nursing homes $(\mathrm{p}=0.097)$ [8].

So, how does life look like for old people who live in nursing institutions? What topics do they pay attention to in their daily life in nursing institutions? Are there other basic topics besides incomes, interpersonal relationship, cognition, and health? When this group of individuals living together for a long time, what culture will be formed?

\section{Methods and Procedures}

This study adopts the method of ethnographic psychology. Ethnographic psychology is a kind of research method based on participatory observation. Participatory observation often takes place in the field, asking questions and having conversations naturally according to context. Therefore, researchers often use informal strategies to work, need to integrate into the group culture, and learn local language. Researchers will initially have a basic and comprehensive understanding to the studied group, preliminarily sort out the possible information source related to the study, and then narrow down the research focus, selecting the suitable research subjects to help understand the life of the studied community.

The researchers of this study entered in participatory observation through the introduction of the director of a nursing home and entered the field as volunteers of counseling and at the same time conducting some research about the psychology of ageing. It took about half a year for the elders to get to know and accept the volunteers and one year to be completely familiar with the volunteers. Researchers became familiar with the elders' language and became part of the community in the nursing home gradually.

After selecting possible research subjects, researchers could interview the subjects and conduct longitudinal study. There were informal interview, semi-structured interview, and autobiographic interview. It took two years to complete this process.

Then, researchers analyzed information collected during participatory observation, interview, and longitudinal study, adopting methods of grounded theory and discoursing analysis. Based on that, researchers extracted and summarized the topics old people care about in their daily lives, discovering the key features of their life world.

\section{Results}

\subsection{Sample Attributes: Analysis of Social Context}

Wuhan is located in the central area of China and between the more economically developed southeast China and less developed northwest China, where southern culture and northern culture interact with each other. For Chinese elders, traditional thinking and modern technology and development coexist in their lives. Researchers conducted their study in a nursing home located in Wuchang District of Wuhan. This nursing home belongs to Hubu community, an old neighborhood composed of four neighborhood committees since 2000 and is next to Yellow Crane Tower and Yangtze Bridge. Hubu community has a total population of 8200 people and 3200 households. There are three art teams including Chu opera group in the community.

\subsection{Results from Participatory Observation}

\subsubsection{The Visible World of the Elders in the Nursing Home}

As an institution mainly providing for the elders, the nursing home is a house with four floors. There is a hall on the first floor after entering the gate, and the elders often watch 
movies, do clapping exercise, or sit about here. A reception room is at the end of the hall. There is an activity room at the left side of the hall, and the elders often play mahjong and card game here in the afternoon. The activity room also has a television, so the elders often watch Chu opera here in the morning. The elders' bedrooms are at the left side of the activity room, and they are mainly double rooms. Some elders with better financial condition will live in single rooms. There are also two medical rooms, one doctors' office, and one massage treatment room on the first floor.

The stairs are at the right side of the hall. Upstairs, there are laundry room and balcony at the corner. People often wash and dry their clothes here, but because its space is relatively small, most elders prefer to use laundry room on the third floor and the rooftop on the fourth floor. The stairs between the second floor and the third floor have paintings hanging on the wall-24 filial piety charts. These paintings mainly serve to remind the staff members in the nursing home of treating the elders with love and filial piety. There are also photos of the elders participating activities in the nursing home, expected to give the elders a sense of home.

The second floor mostly contains old people's bedrooms. Every bedroom has equipment such as television, air conditioner, and water heater. There is also a large special-care bedroom that gather all the elders without self-care ability to care for by caregivers all the day.

Apart from the elders' bedrooms, the third floor has counseling office, office of director of the nursing home, and activity room for painting and calligraphy. Next to activity room for painting and calligraphy, there is a large laundry room. The stairs connecting the rooftop are on one side of the laundry room. When the weather is good, the elders will bask and hang their clothes on the rooftop. Although the building only has four floors, there is an elevator, enabling the elders to go to every floor conveniently. Some volunteers' performances take place on the rooftop sometimes. Standing on the rooftop, the elders can see Yangtze Bridge and other rooftops in the old neighborhood around this building.

Besides that, there is a bungalow on the right side of the building. It provides three meals for the elders in the nursing home and elders living at homes in the community every day. The neighborhood committee is near the nursing home and provides services assisting old people who live at their own homes in the community, such as housekeeping service and mental health counseling.

\subsubsection{Daily Lives of the Elders Living in the Nursing Home}

The nursing home can accommodate 75 elders with complete self-care ability and semi-self-care ability (from first floor to third floor) and 15 elders without self-care ability or with mental dysfunctions (special-care room on the third floor) at most. However, since some old people want to live in single rooms, they will occupy two places. Elders have flexible staying time in the nursing home, and they pay their fee monthly. Due to reasons such as going to hospital, returning their homes, failing to adapt to the life in the nursing home, and passing away, elders do not always stay in the nursing home, and the average number of people living in the nursing home is about 45 per month. From 2014 to now, 181 elders have lived in this nursing home for a period of time. Among them, there are 45 elders stay in long-term, but they often return home for a while during Spring Festival. 37 elders passed away in the nursing home, and 102 elders left the nursing home halfway to return their homes or go to other nursing homes.

The staff members in the nursing home play an important role in the elders' daily lives. They gave the elders family-like social supports. Because China has culture of filial piety, children's supports to their old parents have both material and spiritual meaning. Sending the elders to a good nursing home represents children's material support to elders, but the children's lack of visits to elders will cause the loss of spiritual support, and the elders will be disappointed when comparing their children with others' children. Thus, how to give the elders family-like social support? Caregivers in the nursing home are responsible for daily nursing for the elders, and the volunteer organizations are responsible for giving the elders psychological and emotional care.

The elders in the nursing home are mostly females, and males are only in very small proportion and are mostly uncommunicative. After half a year, the volunteers have become a natural part of the nursing home. The elders have been familiar with the purposes and jobs of the volunteers, and the volunteers have known the basic situations of the nursing home.

The elders have a regular life. They get up and have breakfast at around 7 am every day. After that, elder HML and elder WZZ will practice calligraphy in the activity room on the third floor. In the morning, the oldest man LGT will play CDs of $\mathrm{Chu}$ opera for the elders on television. Later, before watching Chu opera, they will do clapping exercise in the hall on the first floor. They have their lunch at about $11 \mathrm{am}$. After taking a nap, some elders will play mahjong, and some elders such as WLQ and WZZ will play card games. Other elders watch them play. Around $5 \mathrm{pm}$, they will have dinner and then rest for a while. As this is a natural observation, and researchers enter the field as volunteers of counseling, they try not to interfere with the elders' daily lives by interviewing them, unless the elders are familiar enough with the volunteers and show interests of being interviewed. Therefore, over about 2 years, the participatory observation in the nursing home enables researchers to both get to know the elders and conduct interviews and longitudinal study with suitable subjects.

\subsubsection{Daily Lives of the Elders Living at Homes with Assistance of Communities}

As mentioned above, most of old people in China choose to live in their own homes, and there is no exception in Hubu community. According to staff members in the community, Hubu community has 2600 elders over 60 years old. Among them, there are 1730 old people between 65 and 89 and 44 elders over 90 . However, there are only 49 elders (mainly aged above 65) provided for in the nursing home (before May 31, 2018). Suppose the number of elders from Hubu community living in other nursing institutions outside is equal to that of elders from other community living in the nursing home of 
Hubu community, so approximately $97.2 \%$ of the elders are provided for at home. Some of them have either physical dysfunctions or mental dysfunctions, needing assistance services from the community such as sending meals, housekeeping, and mental health caring. Staying at home and watching TV is the main part of their daily lives. Some of them live with their families, while others live alone.

\subsection{Results from Interviews}

\subsubsection{Interviewed Elders}

Over the past two years, researchers have spent 91 days in the field in total. The average working time per day is about 6 hours, so the total working time is about 550 hours. The number of times interviewing each elder is different. HML and MZR are interviewed most frequently, which is 34 times and 27 times respectively, so they are major subjects observed in this study. Other elders are interviewed 2-5 times on average. The interview time is also different every time, based on the elders' activities and arrangements. It is usually around 10 minutes to 1 hour. Interviewed and Analyzed elders are shown in table 1.

Table 1. The elders interviewed and analyzed in this research.

\begin{tabular}{llll}
\hline Code & Sex & Admission Time & Current Situation \\
\hline HML & F & 2015.03 .16 & Left and went to daughter's home in Shanxi (2017.6.23) \\
QXY & F & 2015.07 .07 & Passed away (2017.12.09) \\
GXF & M & 2017.02 .04 & Left to receive therapy (2017.09.07) \\
WCA & M & 2014.07 .20 & Left (2017.12.16) \\
WSX & F & 2014.11 .28 & Live in the nursing home \\
LST & M & 2015.01 .12 & Live in the nursing home \\
MZR & F & 2015.03 .14 & Live in the nursing home \\
WJX & M & 2015.07 .02 & Live in the nursing home \\
XXZ & M & 2016.03 .07 & Live in the nursing home \\
XZY & F & Live in the nursing home \\
YHZ & F & 2017.07 .30 & Live in the nursing home \\
& & Left (2015.5.14) \\
YSM & F & Back again (2016.11.03) \\
YLY & F & Left (2016.12.1) \\
XYE & F & Back again (2017.02.01) \\
DMY & F & Left and back again (2017.02.13) \\
CMJ & M & Left \\
WZZ & F & 2014.12 .30 & Live in the nursing home \\
YJY & F & 2016.06 .28 & Live in the nursing home \\
CCL & F & 2017.08 .15 & Left to receive treatments (2017.12.28) \\
JGY & F & Back again (2018.03.06) \\
ZPP & F & Live in the nursing home \\
DZA & F & Live at home with community assistance \\
\hline
\end{tabular}

\subsubsection{Analysis of Themes in Elders'Daily Lives Based on Grounded Theory}

Since most interviews are informal and few are semi-structured, elders mainly talk about topics relevant to their own daily lives. The materials below are mainly discourses from recordings of interviews (coded with $\mathrm{R}$ as starting), and some of them are also from diaries recorded during field observation on work days (coded with D as starting). Grounded theory is used to analyze content of interviews. Basic coding involves four elements: recordings (R) or diaries (D); date including day, month, and year; name referred to as initials; topics ranked based on the order of interviews. Subsequently, researchers categorize these topics to get several themes, and then analyze the relationships between themes to discover the key features in daily life world of elders with assisted caring.

i Analysis of Life Themes Revealed in Elders' Everyday Conversations

Through basic coding, researchers find themes such as age, health, family relationships, neighborhood relationships, incomes, life feelings, life strategies, and festivals involved in elders' everyday conversations.

$$
\text { a Age }
$$

Many elders tell others their age when they meet someone for the first time. For example, LST, a retired cadre of construction machinery, took out his work certificate to prove that he was born in 1919 when he met the volunteers for the first time; however he was born in 1921 according to his identification card (D-20160321-LST-01). QXY told researchers she was 84 years old when she first met the researchers (D-20160321-QXY-01). YLY said her actual age was 95 when meeting for the first time (D-20160321-YLY-01), and WSX said she was 87 in the first meeting (D-20160321-WSX-01). This is relatively unimaginable for people in other age groups, especially for women.

\section{b Health}

After researchers became familiar enough with the elders in the field, they greeted elders by asking their recent health conditions. Many elders were still willing to talk about their health conditions with researchers even though they were not 
very familiar with the researchers.

For instance, when JGY met the researchers for the second time, she said, "My ears and eyes are not good now. I used to watch the program 'Yangshengtang' which talks about how to stay healthy on TV. (R-20180703-JGY-07)"

When researchers first had a long conversation with WCA, he said that he had many diseases such as hypertension, cerebral infarction and bradycardia although he looked healthy(D-20170603-WCA-02).

c Incomes

YLY told researchers that she had more than 4000 yuan pension monthly when meeting researchers for the first time (D-20160321-YLY-02). WSX told researchers proudly that she had more than 3000 yuan pension monthly:

"He (her second son) treated me well. When I need money, he often gives me 400-500 yuan. He always gives me money when I need some. I have saved much money in Mr. Wang's place. I have saved much. That's how much (she showed five fingers?) (R-20180530-WSX-13)"

\section{d Family Relationships}

Since there are some of her incomes left after paying the fee of nursing home, YLY told researchers she had 4000 yuan pension monthly because she thought she was not the burden of her family (D-20160321-LST-03). She often treats strangers with positive emotions. However, just before the Dragon Boat Festival, researchers saw YLY had a bad mood for the first time. She complained about none of her 4 kids coming to visit her, so she would rather die. She said,

"I was lucky that I pay the fee in the nursing home myself, or why should I even be alive? (R-20160606-YLY-0)"

QXY's son died because of cancer when he was 45 , and QXY's husband died at the beginning of 2017. She said, "My husband died. My son also died. There is no meaning of staying alive for me(R-20170831-01)." Half a year later, according to WSX, QXY had a disagreement with her daughter, so she took excessive sleeping pills she collected since long ago and passed away (D-20180114-WSX-01).

On the positive side, JGY said proudly,

"I rely on him (her son). All the people in my community say he is good.(R-20180703-JGY-06)"

"What do I think about now? I don't want other things. The only thing I need is peace. My sons and grandsons are all very good. (R-20180703-JGY-07)"

MZR talked about the dilemma of old people's living mode,

"I am very ambivalent, very ambivalent. I really want to live with my son and daughter, but I think I will affect their work. (R-20170617-MZR-05)"

e Neighborhood Relationships

Doctor Chen's mother has lived in the nursing home for nine months, but she said her mother knew almost no one there. She said,

"She (Doctor Chen's mother) does not want to communicate with other people. She would rather be alone. Also, it depends (on who she is communicating with). (R-20180314-CYL-07)"

WCA has lived in the nursing home for three years, but he said,
"I have not spoken a word to many men in the nursing home so far. (R-20170725-WCA-10)"

f Life Feelings

In the nursing home, besides special activities and guests coming, it is quiet for most of the time. As mentioned above in the neighborhood relationships, many elders do not talk with each other when sitting together. They sit quietly for most of the time, as if they are waiting for something. MZR said,

"We are happy for the coming of you guys. If you guys do not come, our lives are boring and monotonous. When you guys come, our lives suddenly become lively. (R-20170617-MZR-03)"

One day, HML talked a lot with the researchers in front of the activity room, and raised a question,

"I don't want to live long ago. But what can I do? (D-20160411-HML-01)"

WZZ had a similar statement as HML on this question. After doing lots of activities one day, she sighed,

"How can I spend my time otherwise? (D-20160411-WZZ-01)"

\section{g Life Strategies}

WCA thought that old people face changes and adaptations after retirement, or they cannot get used to their later lives:

"Some people change slowly, and maybe they cannot get used to their lives after three or five years...It's better for them to try more, adjust, and adapt based on their own situations. (R-20170725-WCA-07)"

As her body becoming weaker, JGY reduced the frequency of her physical activities to lower the risks of falling down, changing her way of exercising:

"I was better last year. I could still walk around outside. I can't this year. My ability falls short of my wishes, and it takes me a long time to walk. It will be troublesome if I fall down or bump into someone outside. I simply don't walk later. I can't walk either. So I stay at home. If the weather is good, I will do whole body massage myself. (R-20180703-JGY-06)"

ii. Key Features of Life World of Elders with Assisted Caring: Live a Good Life

After analyzing the life themes of these elders, researchers find that despite various topics in the conversations and their different personalities, the themes all indicate a key point: how to live a good life.

a Living in Nursing Home to Adapt to Frailty in Body

For example, when CMQ was talking about how his body got worse this year, he started with how he had meals.

"My appetite has reduced now, reduced a lot. I am gradually getting older, my digestion is getting worse (his voice dropped). (R-20180701-CMQ-02)"

$\mathrm{He}$ also mentioned his family relationships and the reason why he went to the nursing home from talking about how his body got worse:

"My kids all had their own businesses, and I lived alone at home. I came here in October...I could not have meals at home alone. I lived on the fifth floor, so it's very tired for me to walk up and down the stairs. I live on the second floor here because there is no vacancy on the first floor. I said yes. It's okay for me to live on the second floor. 


\section{(R-20180701-CMQ-07)"}

$\mathrm{He}$ mainly talked about how they have meals when mentioning his life in the nursing home.

"It's good here. They send all three meals to your hands. You don't need to move, and they wash your clothes for you. If you don't like the food here, you can eat elsewhere. I eat outside sometimes (raised his voice). There are so many people. Not everyone finds it suitable for the appetite. Not everyone likes the food here! I have lived here for three months, and I have eaten outside three times. (R-20180701-CMQ-08)"

b The Life in the Nursing Home Continues the Life at Home.

YLY said she would rather die if none of her kids came to visit her. On the opposite side, she was saying she had reasons to live if her kids visited her. In fact, her kids visit her frequently. One day, when she was complaining about her kids not visiting her, her kids came to the nursing home just in time and brought her some oranges (she seldom asks her kids to visit her on phone). She was very happy (D-20161112-YLY-01). Therefore, her complaints sometimes bring her the motive of staying alive.

Even though MZR is in the nursing home, she cares about her kids very much.

"We are here (the nursing home), so they (kids) can have less troubles. (R-20170617-MZR-04)"

c Keeping positive to live a Good Life

WCA adapts his life after retirement positively to prevent illnesses and to live a better life:

"If you are withdrawn and cannot communicate with others easily, and you cannot adapt to the life after retirement, you will be very depressed and lonely. If it lasts long, you may even suffer from diseases because of that. (R-20170725-WCA-07)"

\section{d Longevity Is a Blessing}

When talking about age, the elders never conceal their real age because they are too old, and they even give a bigger number on their age. Researchers initially did not get the reason why LST showed his work certificate as mentioned above. In fact, he wanted to prove that he is actually older than that because his birth date on his identity card is 1921. YLY told researchers directly that her age is 95 , but the age indicated in her identity card is smaller, which is 90 . HML also said that her age on her identity card is smaller because she went to school late. She was born in 1921, but her identity card showed it was 1926 (D-20160411-HML-07).

The elders like to give a bigger number on their age not only because they want to get subsidies for their advanced age, but also because Chinese consider longevity as a blessing. Advanced age represents success of life. For instance, HML took researchers to her room to see her calligraphy works when they met for the second time. She also taught researchers how to write the Chinese character for longevity (D-20160321-HML-02).

\subsection{Analysis of Elders' Life World Besides Conversations}

The communications in the nursing home include language, music, movement, and silence. Silence is the main communication way among the elders. WJX has lived in a room for two years, but he said he did not know anyone in the room, because he did not know anyone's name (D-20180714-WJX-24).

XZY almost cannot hear others' conversations so that researchers thought she was unwilling to talk and preferring to live in a silent world. Until one day, she felt like she knew the researchers, so she greeted the researchers warmly and talked to them, though she did not know what others are talking about. She said,

"Every day of life counts...How many years can I still live now? (R-20180704-XZY-12)"

More positive expression may be what JGY said,

"Every day I live is a happy day, a joyful day. The living conditions now are good. (R-20180703-JGY-07)"

This is the attitude of elders with assisted caring living in silence and waiting every day. This is the expression of life meaning from these elders. This also gives the elders the motive of staying alive. Although silence may seem lonely, it may also be peace; Death is waiting for them, but there is still hope. Tao always likes to sit quietly in the hall, and this seems a bit cold for others. When being asked, she said she did not like to talk, and she liked quietness (D-20161220-T). The eldest daughter of WSX promised to take her on a trip when she was 90. This is this hope of WSX's life.

"She (eldest daughter) said she will take me to live with her next year when I am 90. She will first take me to Beijing. After traveling in Beijing for a while, we will go to other places. (R-20180513-WSX-13)" "My daughter will come here to pick me up, to take me to her place. She wants me. We will go to Beijing first to see Chairman Mao's picture and flag-raising. Chairman Mao's picture can be risen. Do you know that? (R-20180513-WSX-14)"

Elders with assisted caring, either, talk about topics basically related to age, health, family relationships, neighborhood relationships, incomes, life feelings, and life strategies. In the life world of these elders, some of them feel happy and peaceful, and some of them feel sad and depressed. Most of them are quiet and waiting. Their lives both have positive and negative sides. But regardless of their concerns to their health conditions or complaints about their relatives or neighbors, they want to stay alive better, including those living in nursing home to adapt to frailty in body, the life in the nursing home is a continuous form of the life at home, keeping positive to live a good Life and believing that longevity is a blessing.

\section{Conclusion}

Apart from incomes, interpersonal relationships, cognitive abilities and health, the most fundamental topic for the elders is how to live. Although most elders in the nursing home are aged, they still hope to live longer; on the basis of longer life, they then will hope to live happier. Staying alive is the basis, and living happier is a higher pursuit for the elders.

The nursing homes functions as the "homes" for the elders. Although factors such as health, cognitive abilities, life 
satisfaction are crucial, overcoming various difficulties of living is a more important subject.

"Live a day, live a happy day" is a positive expression on this subject. From a negative viewpoint, they are counting the days in life: "Live a day, count it as a day." Even though, in the Chinese culture, longevity is still good to pursue: longevity is a blessing---staying alive is indeed meaningful, no matter it is happiness or misery.

\section{Discussion}

The depictions of lives of elders with assisted caring are not involved in the job of academic research mostly, especially for psychology based on quantitative research. Therefore, there are very few articles studying their life world from academic perspective in many journals of academic studies. The written materials about their life world are seen in the newspaper occasionally. For instance, Seaver [9] recorded her life world in a nursing home from the inside on Newsweek:

You see, I'm old. And, I'm not as healthy as I used to be. I'm not necessarily happy with it but I accept it. Occasionally, a member of my family will stop in to see me. He or she will bring me some flowers or a little present, maybe a set of slippers -- I've got 8 pair. We'll visit for awhile and then they will return to the outside world and I'll be alone again.

Of course, there are some academic studies about the lonely life world of elders living alone at home occasionally. One study analyzed descriptive phenomenological studies with older women ( $\mathrm{N}=81$, aged 75-98), and found a 4-level taxonomy of life-world [10]. Hope emerges from one's past, through one's present and toward a horizon that has various degrees of clarity. Although older women live alone, suffering with chronic pain or other health related situations, hope is enacted in contexts apart from terminal illness. Unfortunately, the study did not draw much attention from peers so far, and it has not been cited for once so far.

That is to say, for the aged people in the nursing homes, how to live is the most basic life theme in their eyes; the so-called "successful aging" [11] is only a pursuit of higher life goals in the elders' world. If the researchers still focus only on the negative influences of environmental cues towards aging [12], the positive effect of motivated cognition during aging [13], from successful aging to esteem of the fourth age [14] instead of understanding their loneliness and the strong desire of living a longer life, which therefore separates the mainstream world from the elders. Even under the Chinese culture that puts a high value on providing for the elders, there still is such deviation in research. There are very few documents about the life world of the elders in China. Only one academic study about cellphones in Chinese rural elders' life world could be found on the Internet [15]. It sorts out how rural elders use cellphone, what kind of cellphones they use, and their time for using cellphones to understand the basic situation of rural elders using cellphones. It also reveals that young people in current society have more advantages in knowledge and technology and highlights the existence of generation gap. New technology facilitates the social inclusions of young individuals but excludes words from the elders. In this article about elders with assisted caring, elders are also in the status of losing words and being isolated. However, their hope of life and pursuit of life meaning are still worth understanding and studying.

\section{References}

[1] United Nations. Political Announcement in Madrid, adopted from http://www. un.org/chinese/esa/ageing/declaration. htm. 2002-04-12) [2016-11-19].

[2] J. Melorose, R. Perroy, and S. Careas. World Population Prospects: The 2015 Revision, Key Findings and Advance Tables. In "Working Paper No. ESA/P/WP. 241, 2015 (pp. 1-59)".

[3] T. Wang and C. Hou."A Topical Structure Analysis on Chinese Aging Study and Practice in the View of Culture Psychology". In Proceedings of the Second Summit Forum of China's Cultural Psychology, T. Xie, L. Hale, and J. Zhang, Eds. America: The American Scholars Press, 2016, (pp. 164-170).

[4] K. Shen. (2013) Change of Chinese elders living mode. Beijing: Social Science Literature Press, pp. 18, 20, 92-123,”

[5] Ministry of Civil Affairs of the People's Republic of China, (2017a)The statistic report of social service development in 2016, adopted from

http://www.mca.gov.cn/article/sj/tjgb/201708/2017081500538 2.shtml [2017-08-03]

[6] Ministry of Civil Affairs of the People's Republic of China, (2017b). Annual Statistic: data of social service in China. Chinese Statistic Press.

[7] Y. Zeng. (2010) A study on the elders' family, health and caring cost. Beijing: science press, P172, 176.

[8] X. Lin and Y. Liu. (2015) Compare on the life quality of the elders between living home and nursing home in the urban. Jinan: Shandong People Press, pp91-95.

[9] A. M. Seaver. (1994) My world now. life in a nursing home, from the inside. Newsweek, 123(26), 11.

[10] Porter E. J., T. O. Oyesanya, and K. A. Johnson (2013). Hoping to see the future I prefer: an element of life-world for older women living alone. Ans Adv Nurs Sci, 36(1), 26-41.

[11] Baltes, P. B. and J. Smith, (2003) New frontiers in the future of aging: from successful aging of the young old to the dilemmas of the fourth age. Gerontology 49 123-135.

[12] Baltes, M. M. and L. L. Carstensen (1996). The process of successful ageing. Ageing And Society 16, 397-422.

[13] Mather, M. and L. L. Carstensen (2005). Aging and motivated cognition: the positivity effect in attention and memory. Trends in Cognitive Sciences 9, 496-502.

[14] Hsu, L. M., J. Chung, and E. J. Langer (2010). The influence of age-related cues on health and longevity. Perspectives on Psychological Science A Journal of the Association for Psychological Science 5, 632.

[15] W. Wu. (2016) Cell phone in the life world of the elders living in the countryside. (Master dissertation, Centre China Normal University). 